\title{
BMJ Open Protocol for a systematic review of research on HPV and cervical cancer in Ghana, up until the year 2017: informing research and policy direction on cervical cancer prevention in Ghana
}

\author{
Adolf Kofi Awua, Edna Dzifa Doe
}

To cite: Awua AK, Doe ED Protocol for a systematic review of research on HPV and cervical cancer in Ghana, up until the year 2017: informing research and policy direction on cervical cancer prevention in Ghana. BMJ Open 2018;8:e020183. doi:10.1136/ bmjopen-2017-020183

- Prepublication history for this paper is available online. To view these files, please visit the journal online (http://dx.doi org/10.1136/bmjopen-2017020183).

Received 19 0ctober 2017 Revised 14 May 2018 Accepted 8 June 2018

Check for updates

(C) Author(s) (or their employer(s)) 2018. Re-use permitted under CC BY-NC. No commercial re-use. See rights and permissions. Published by BMJ.

Cellular and Clinical Research Centre, Radiological and Medical Sciences Research Institute, Ghana Atomic Energy Commission, Accra, Ghana

Correspondence to Dr. Adolf Kofi Awua; a_awua@yahoo.com

\section{ABSTRACT}

Introduction For a country that lacks a national cervical cancer screening/prevention programme, there is the need to assess the volume of country-specific information, and the status of research on HPV and cervical cancer, in order to provide evidence that will inform policy and further research. The aim of this protocol is to plan an intended systematic review, which is to identify research gaps, prevent unnecessary duplication of work and enable collaboration.

Methods and analysis This protocol, developed according to the Preferred Reporting Items for Systematic Review and Meta-Analysis Protocols statement and registered by PROSPERO (CRD42017075583), will apply a 13-point eligibility criteria to screening and selecting peerreviewed research articles and grey literature. These will be obtained from searches in databases, including, among others, those of the National Centre for Biotechnology Information, Cochrane Central Register of Controlled Trials, Google Scholar and the digital collections database of research publications of Universities in Ghana. Collected data will be aggregated and summarised according to emerging themes and simple descriptive statistics. Ethics and dissemination The study will use publicly available data and will not identify authors of the publication by name. In light of these and as has been indicted, research ethics clearance is not required for evidence syntheses in such reviews. The review will be published in peer-reviewed scientific journals and presented at local and internal conferences as the opportunity becomes available.

PROSPERO registration number CRD42017075583.

\section{INTRODUCTION}

\section{Rationale}

Cervical cancer remains a major global public health concern, with a global estimate in the year 2012 of 527624 new cases and 265653 deaths due to cervical cancer. ${ }^{1-3}$ Specifically, Africa continuously bears a highly disproportionate, high burden of the incidence and mortality of cervical cancer. This is evident by the 92000 new cases and 60098 deaths
Strengths and limitations of this study

- The comprehensive search and screening for eligibility of and the inclusion of both abstracts and full-text literature, whichever is available, is a major strength of this protocol.

- Additionally, this protocol is designed strictly according to the guidance of a globally accepted guideline (Preferred Reporting Items for Systematic Review and Meta-Analysis Protocols) for the development of a systematic review protocol.

- The exclusion of literature, not directly as a result of research, due to the scope implied by the title, potentially may result in loss of some insightful literature.

- The protocol may limit the inclusion of some publication not indexed in the databases to be searched.

estimated to have occurred in the year 2012 in Africa. ${ }^{14}$ In Ghana, it is one of the two most prevalent (the other is breast cancer) cancers and causes of cancer death. Furthermore, with an estimated 1.6 million women at risk, 3052 cases were estimated to have occurred in 2012. ${ }^{1}$ These high burdens of cervical cancer necessitate the development and implementation of a cervical cancer prevention and control plan and/or programme for Ghana. In order to do this, pieces of evidence from research are very useful.

All around the world, national and regional cervical cancer prevention and control programmes, and clinical management guidelines on human Papillomavirus (HPV) infection and cervical cancer are all informed and reviewed mainly by synthesising information from the findings of all kinds of studies on HPV infection, cervical pre-cancers, cervical cancer and other HPV-related diseases. ${ }^{5-9}$ These syntheses may be by summary literature reviews or/and by systematic reviews of 
studies, ranging from laboratory-based studies (cellular and molecular biology) through observational studies (prevention, early detection and treatment), interventional studies (including those on education, early detection, vaccination and treatment), studies on survival and palliative care to modelling and economic assessment studies. ${ }^{10-13}$

The use of systematic reviews for such purposes is clearly shown in the development of the WHO guideline for cervical cancer prevention and HPV screening in low-income countries, known as 'the Pink Book'. This is a major cervical cancer prevention plan that was based on extensive systematic review of different kinds of studies from different populations. ${ }^{14}$ A more recent example of the review of a national cervical cancer screening programme, which was informed by the analysis of information obtained by a literature search of existing publications, was reported for Australia. ${ }^{15}$ Specifically, the recommendations for the review of the programme were put forward using data on the following: HPV transmission, natural history of HPV and cervical cancer, cervical screening and vaccination programmes, and a modelling and economic assessment study. These formed the bases for the transition from a 2-year interval of cytology-based national cervical screening to a 5-year interval of primary HPV screening with partial genotyping. Additionally, South Africa's cancer guideline were also informed by literature reviews of relevant studies in South Africa and from other countries. Such summary reviews, systematic reviews, meta-analysis and re-analysis are performed both with national and international data in order to have a globally informed policy direction for each country.

For Ghana, a country that lacks a clinical guideline for HPV and cervical cancer treatment, a national population-based cancer registry and a national cervical cancer screening/prevention programme, there is the need to assess the volume of country-specific information and the status of research on HPV and cervical cancer in order to provide evidence that will inform policy and further research. This will lead to identifying research areas that have very limited data and those that are being overstudied. Furthermore, the findings of such a review will serve as a guide to direct current research and encourage new HPV and cervical cancer researchers in Ghana.

The review intended with this protocol hopes to achieve this by throwing light on the information/evidence gaps in the spectrum of information needed to inform policy. Additionally, this will ensure the judicious use of limited research funds/resources and enhance complementary collaboration. Overall, these will be useful for a comprehensive cervical cancer prevention planning and the development of guidelines and prevention programmes. To this end, this protocol was therefore designed with the aim of providing the plan and guidance for the performance of an intended systematic review. The intended systematic review has the under-listed as its specific objectives.

\section{Objectives of the planned systematic review}

1. Quantify the volume of research on HPV and cervical cancer in Ghana.

2. Identify HPV and cervical cancer research areas currently being studied in Ghana.

3. Collate the major findings and recommendations presented in publications on HPV and cervical cancer, from studies conducted in Ghana up until the year 2017.

4. Determine the proportion of both internal (inter-institutional, intra-institutional, inter-researcher in Ghana) and external (international) research collaborations.

5. Present an overview of the human resource involved with HPV and cervical cancer research in Ghana.

\section{METHODS}

\section{Patient and public involvement}

Patients and the public are not involved in this study.

\section{Guidelines and registration}

This protocol was developed with guidance in accordance to the Preferred Reporting Items for Systematic Review and Meta-Analysis Protocols (PRISMA-P) statement, and the review that is to be guided by this protocol will be carried out in accordance with PRISMA guidelines. The protocol was registered with the International Prospective Register of Systematic Reviews (PROSPERO) in 2017 and has been assigned the identification number CRD42017075583. The full documentation is available online (http://www.crd.york.ac.uk/PROSPERO/ display_record.php?ID=CRD42017075583).

\section{Eligibility criteria}

In selecting the relevant studies, the following criteria (inclusion and exclusion) will be used:

1. POPULATION: Studies having a study location or location of sample collection in Ghana, and/or study population of Ghanaians or persons living in Ghana, will be eligible.

2. INTERVENTION: No limitation on intervention was applied and non-intervention studies will be eligible.

3. CONTEXT: Although the primary focus was on cervical cancer, studies of HPV infection in other cancers will be considered eligible.

4. OBJECTIVE/OUTCOME: Studies with objectives related to and providing information on primary, secondary and tertiary prevention activities of cervical cancer, the epidemiology and biology of HPV, cervical lesions and cervical cancer and other HPV-related cancers will be eligible.

5. STUDY DESIGN: No limitation is placed on study design, rather it will be a variable of interest.

6. Studies with published or available unpublished data made as of December 2017 will be eligible.

7. No language limitations will be applied.

8. Multi-country studies for which data on Ghana were not separately presented will be ineligible. 
9. Research reports published by a research team or as a result of a research activity will be eligible.

10. Thesis of research study on cervical cancer, HPV and HPV-related cancers published on the websites of universities in Ghana will be eligible. However, if the information are available in peer-reviewed publication, the information from the thesis will not be used. However, the thesis will be counted as one of the thesis publications in Ghana on the subject of interest.

11. Conference presentation or abstract for which a fulltext article is not available shall be eligible.

12. All grey literature for which a full-text article is available shall be eligible.

13. Government publications and non-governmental organisation (NGO) publications, policy documents and other such publication are not eligible as long as they are not a direct publication related to research. As implied by the title "...systematic review of research...", the protocol is for a review of research, therefore policy documents with no direct relation to research in Ghana cannot be included. However, for those for which there is a direct link with research in Ghana, if published peer-reviewed articles are included, then these will not be included. Furthermore, to the best of our knowledge, there is only one such NGO publication, which has most of its information published in different research articles by the collaborating researchers on that project.

\section{Information sources}

Online search for each type of publication in the following databases will be conducted in the month of February 2018. Peer-reviewed research articles will be searched for in all databases of the National Centre for Biotechnology Information (www.ncbi.nlm.nih.gov/), Embase, CINAHL and Google Scholar. Reference lists of included studies will be also scanned to identify additional relevant publications. Clinical trials will be searched for in the Cochrane Central Register of Controlled Trials (CENTRAL), ClinicalTrials. gov and the WHO International Clinical Trials Registry Platform. The specific grey literature, thesis, researchbased reports, conference presentations and abstracts will be searched for both in Google search engine (www. google.com), Google scholar (www.scholar.google.com) and in the digital collections database of research publications of the University of Ghana (UGSpace; http:// ugspace.ug.edu.gh/), the Kwame Nkrumah University of Science and Technology (KNUSTSpace; http://ir.knust. edu.gh/) and the University of Cape Coast. These are the leading public universities in Ghana.

\section{Search strategy}

The search strategies will involve searching within the search fields Titles and Abstracts, using the same terms/ keywords across the different databases and website to be searched. It must be noted that the search terms will be appropriately entered according to the requirement of each of the databases to be searched. The search strategy for PubMed (presented below) will be modified to include the specific truncation, wild cards and other functionalities required for each database. In other words, this strategy will be translated to the equivalent search strategy for each of the stated databases, taking into considerations the databases' specific terms and qualifiers, truncation commands (eg, *, \$), wildcards (eg, ?, \#), proximity operators (adj) and phrase searching ("”) requirements. A filter by year (up to 2017) will be applied for the search in each database. For each database or website searched, the related search term and the number of records retrieved and/or screened will be recorded.

As an example, the search for peer-reviewed literature in PubMed will be conducted with the following search strategy.

Ghana* AND Papillomavirus OR Papilloma virus OR HPV OR Pap test* OR Cervical cancer screening OR Pap smear OR HPV test* OR VIA OR VILI OR Visual inspection OR self-sampling OR self-collection OR Colposcopy OR Cryotherapy OR Cervical lesion OR Cervical intraepithelial neoplasia OR CIN 1 OR CIN 2 OR CIN 3 OR Cervical dysplasia OR 'Low-grade Squamous intraepithelial lesion' OR LSIL OR HSIL OR Genital warts OR cervical cancer, 'cancer of the uterine cervix' OR cervical carcinoma OR Head-and-Neck cancer OR HNSCC OR Anal cancer OR Oropharyngeal OR Oral cancer OR Throat cancer OR Vaginal cancer OR Vulva cancer OR Ovarian cancer.

In order to reduce publication bias and for the fact that some hospital data on this subject in Ghana are presented in conference abstracts, grey literature will be searched. This will be done using the same search terms as indicated for the PubMed search, with Boolean commands, and additional modification as may apply to the Google search engine and targeted website or database to be searched. The grey literature search strategy will be similar to those often described for grey literature, ${ }^{57}$ but with the following modification. The search results will be reviewed by the title and the short text description that will be associated with the title and the selected links followed.

\section{STUDY RECORDS}

\section{Data management}

Database search results of the two independent researchers (AKA and EDD) will be saved in their respective database user accounts. Citation records (search results) of the identified titles and abstracts will be merged and managed in the reference manager Zotero, using a single online account, which will be linked to the stand-alone accounts on the personal computers of each of the independent researchers (AKA and EDD).

Searches for grey literature will be managed as follows: the link to each potential relevant search result, among the first 100 records retrieved, will be followed and bookmarked in a new tab of the search browser. The web address will be exported to the Zotero reference manager. Each of the bookmarked, potential relevant results will be further screened for selection or otherwise, according to 
the stated eligibility criteria. Selected relevant records will be downloaded or their bookmarks maintained.

Any disagreement between the two independent researchers will be resolved in an open discussion. However, if an agreement is not reached, a third independent reviewer will resolve the disagreement. Fulltext documents or abstracts will be downloaded and arranged in a directory according to the type of publication (peer-reviewed article, thesis, book of abstracts etc). Extracted data will be managed in Nvivo and SPSS V.20.0.

\section{Selection process}

Each search result will be screened following a stepwise process. The titles of the resulting peer-reviewed articles, theses and/or abstracts will be reviewed, and if selected, their records will be saved in the researcher's account for each database searched. The citation records will thereafter be exported to a reference manager. The merged records of the two researchers in the reference manager will be assessed and duplicates removed. Abstracts of each record will be screened by applying the eligibility criteria and the relevant ones noted. For records that have full-text documents, these will be further screened, applying the criteria of eligibility. The selected relevant studies, both those that have full text and those with only abstracts, will be included in the review. For grey literature search and searches of website, search results will be reviewed by titles and the short descriptions associated with each. The website of each selected search result will be bookmarked and the web address exported to a reference manager. These records will be further screened, applying the criteria of eligibility, and relevant records included the review. Each of the researchers (AAK and EDD) will independently perform these activities. All disagreements (including those related to merging, screening and selection of search results) will be resolved in an open discussion between the two researchers. Unresolved disagreement will be resolved by a third independent reviewer to be agreed on by the two independent researchers.

\section{Data collection process}

Using the Data Collection Tool 1, data will be extracted from the full-text documents, abstracts and webpages. These will include Title, author initials, year of publication, year study was conducted, type of publication, study location, type of study, target population, setting of the study, ethical considerations, major research area, major findings, and major recommendations for research and policy. If need be, authors will be contacted for further clarification or additional information using the email provided on the articles or by a profile search in the Google search engine.

\section{Major data items/elements}

Major findings or data elements will include the following:

1. Source of funding and support other than funding.

2. Proportion of studies that involved internal and external collaboration.
3. Proportion of studies that reported on ethical conduct.

4. The major researched areas: biological, primary prevention, secondary prevention and tertiary prevention.

5. Quantitative data: age at cervical cancer diagnosis; age-specific cervical cancer and lesion prevalence; age-specific HPV prevalence (indicate study population); awareness of cervical cancer, risk factors, cervical cancer type, symptom incidence and prevalence; diagnosis and treatment (approaches, option, stage and outcomes); factors associated with cervical cancer; HPV genotype prevalence (indicate study population); HPV infection type prevalence (indicate study population); overall HPV prevalence (indicate study population); HPV risk type prevalence (indicate study population); knowledge of any issues and its association; modelling prevention; other HPV-related cancer prevalence; perception (cause, risk factors etc); quality of life; screening history/rate; screening strategies and preferences; and vaccination.

6. Qualitative data: approach to cervical cancer prevention (hospital-based screening, community-based screening activities); barrier to screening (individual, national, cost); educational strategy employed (community-based education, professional group, religious group, mode of education, type of education materials); facilitators of screening and vaccination (cultural, financial, religious, personal, family, social).

7. Major recommendations for research and policy.

\section{EXPECTED OUTCOME}

\section{Major outcomes}

One of the major outcomes of the intended review is whether the full spectrum of HPV and cervical cancer research, needed to inform policy in a country, are being conducted among the population of women in Ghana. The taxonomy of these researches shall be as follows:

a. Biological Research: HPV biology, genome analysis, cancer cell biology, cervical cancer biomarker.

b. Primary Prevention Research: group education, health professional training, knowledge, attitude and perception, risk group assessment, risk factor assessment, psychosocial factors and health seeking behaviour, vaccination.

c. Secondary Prevention Research: prevalence of HPV/ HPV screening, Pap screening/prevalence cervical lesions or cancer, VIA screening, co-testing, factors influencing screening attendance.

d. Tertiary Prevention/Clinical Research: diagnosis, reporting, clinical manifestation, treatment, quality of life, palliative care, incidence/survival/mortality studies, cancer registry data, factors influencing treatment seeking.

Specifically, the imbalance in the focus of researchers in respect of these research areas will be identified. Furthermore, the volume of country-specific information on each of these research areas will be an outcome of interest. Having done these, the country-specific information gap, 
which needs to be filled to enable the development of a comprehensive policy for cervical cancer prevention programme in Ghana, should become clear.

In a nutshell, information that will help focus research, foster collaboration, enhance the identification of research niches and attract young researchers to the fields of HPV and cervical cancer research are expected to be generated.

\section{Risk of bias and quality assessment of individual studies}

Quality of each publication will be assessed independently by the two researchers (AKA and EDD), using one of three quality tools, as will be appropriate. These include the Effective Public Health Practice Project Quality Assessment Tool for Quantitative Studies (intervention studies), the US National Institutes of Health's National Heart Lung and Blood Institute Quality Assessment Tool for Observational Cohort and Cross-Sectional Studies, and the Critical Appraisal Skills Programme Qualitative Research Checklist. Generally, these tools assess how clearly the aims and relevance of a study are indicated, the study design used, the extent of the identification of confounders, the participant representativeness of the study population and the data collection methods used. In addition to these key indicators of quality of a publication, each of the tools was modified by the addition of seven questions to assess the quality of research ethics conduct and reporting for each publication. A 'Yes', 'No' or 'Not reported' response will be accorded, which will be weighted on a scale of 3,2 , and 1 , respectively. These will be independently performed by the two researchers (AKA and EDD). An overall average for each article will be determined and ranked as either good, fair or poor, based on the same scale, respectively. The distribution of these quality indicators will be reported in the review. However, in order to reduce the potential of publication bias and achieve a comprehensive review, and in light of the fact that the volume of research on cervical cancer and related research areas in Ghana is not high, records will not be excluded based on quality assessment results.

However, bias may arise when aggregating information from studies that are dissimilar in respect of study population, study type and study design. This potential bias will be avoided by not performing meta-analysis and by reporting the differences in the studies included or the specific information extracted to define the limits thereof. Rather, ranges for quantitative information will be reported.

In view of variability in information to be retrieved (particularly of grey literature) and the potential of bias, search results will be included if the information they provide is considered relevant and complete enough to help inform future research and policy on cervical and other HPV-related cancers in Ghana.

\section{Data synthesis}

In respect of the information to be included in the review, data on the research areas will be summarised in a tabulation form, which will present the frequency of publications, total number of researchers participating in the identified areas of cervical cancer and HPV research, and the level of consistence of publication (year-on-year) in the area. A graphical plot of the total number of publications and number of each type of publication over the study period will be generated.

In respect of quantitative information/findings, no meta-analysis is to be performed for the intended review; however, these data will be reported as ranges, alongside a mode. Non-quantitative or descriptive and qualitative information/findings will be summarised using the thematic synthesis approach; this involves generating codes for identified major findings 'line-by-line', developing 'descriptive themes' from the codes and then generating 'analytical themes'. ${ }^{9}$ Specifically, the major findings of the studies will be extracted verbatim into MS Excel software, such that each major finding will be placed in a single cell of the same column. The two researchers will independently code (with a short phrase(s)) each of the findings, based on its content and meaning, and place the initial descriptive code(s) in the adjacent cell, in the next column. These codes will be reviewed (by AKA and EDD), and based on difference and similarities, these will either be merged to form new codes or categorised as subcategories of a code. The independent researchers will agree on the final descriptive codes. These will constitute the descriptive themes. The analytic theme will then be generated by developing concepts or formulating an interpretation or hypothesis from the findings within each descriptive theme. The same will be done for the extracted recommendations for policy and research.

Contributors AKA made substantial contributions to conception and design of the protocol. EDD and AKA were involved in the drafting of the protocol, the crosschecking with the PRISMA-P checklist and in revising the protocol critically for important intellectual content. Both authors have given final approval of the version to be published. Both authors agreed to be accountable for all aspects of the work in ensuring that questions related to the accuracy or integrity of any part of the work are appropriately investigated and resolved.

Funding This research received no specific grant from any funding agency in the public, commercial or not-for-profit sectors.

Competing interests None declared.

Patient consent Not required.

Provenance and peer review Not commissioned; externally peer reviewed. Author note This protocol is not an amendment of a previous protocol.

Open access This is an open access article distributed in accordance with the Creative Commons Attribution Non Commercial (CC BY-NC 4.0) license, which permits others to distribute, remix, adapt, build upon this work non-commercially, and license their derivative works on different terms, provided the original work is properly cited, appropriate credit is given, any changes made indicated, and the use is non-commercial. See:@http://creativecommons.org/licenses/by-nc/4.0/.

\section{REFERENCES}

1. GLOBOCAN, IARC. GLOBOCAN 2012: Estimated cancer incidence, mortality and prevalence worldwide in 2012; Fact Sheets. 2012 http://globocan.iarc.fr/factsheet.asp.

2. Jemal A, Bray F, Center MM, et al. Global cancer statistics. CA Cancer J Clin 2011;61:69-90.

3. Ferlay J, Shin HR, Bray F, et al. Estimates of worldwide burden of cancer in 2008: GLOBOCAN 2008. Int J Cancer 2010;127:2893-917. 
4. Jemal A, Bray F, Forman D, et al. Cancer burden in Africa and opportunities for prevention. Cancer 2012;118:4372-84.

5. Adams J, Hillier-Brown FC, Moore HJ, et al. Searching and synthesising 'grey literature' and 'grey information' in public health: critical reflections on three case studies. Syst Rev 2016;5.

6. Olson B, Gribble B, Dias J, et al. Cervical cancer screening programs and guidelines in low- and middle-income countries. Int J Gynaecol Obstet 2016;134:239-46.

7. Godin K, Stapleton J, Kirkpatrick SI, et al. Applying systematic review search methods to the grey literature: a case study examining guidelines for school-based breakfast programs in Canada. Syst Rev 2015;4:138

8. Arbyn M. European Commission. Directorate-General Health \& Consumer Protection. European guidelines for quality assurance in cervical cancer screening. Luxembourg: Office for Official Publications of the European Communities, 2008.

9. Thomas J, Harden A. Methods for the thematic synthesis of qualitative research in systematic reviews. BMC Med Res Methodol 2008;8:45.

10. Dickinson J, Tsakonas E, Conner Gorber S, et al. Recommendations on screening for cervical cancer. CMAJ 2013;185:35-45.
11. Saslow D, Solomon D, Lawson HW, et al. American Cancer Society, American Society for Colposcopy and Cervical Pathology, and American Society for Clinical Pathology screening guidelines for the prevention and early detection of cervical cancer. J Low Genit Tract Dis 2012;16:175-204.

12. Money DM, Roy M, Scrivener J, et al. Canadian consensus guidelines on human papillomavirus. J Obstet Can 2007;29:S1.

13. Saslow D, Castle PE, Cox JT, et al. American Cancer Society Guideline for human papillomavirus (HPV) vaccine use to prevent cervical cancer and its precursors. CA Cancer J Clin 2007;57:7-28.

14. WHO. Guideline Development Group. Comprehensive cervical cancer control: a guide to essential practice. 2nd edn. Geneva: World Health Organization, 2014.

15. Lew JB, Simms KT, Smith MA, et al. Primary HPV testing versus cytology-based cervical screening in women in Australia vaccinated for HPV and unvaccinated: effectiveness and economic assessment for the National Cervical Screening Program. Lancet Public Health 2017;2:e96-107. 